\title{
Un dernier regard...
}

\section{A last glance...}

\section{G. Amarenco}

C) Springer-Verlag France 2011

La vie n'est sans doute qu'un éternel recommencement..., car vous lisez le dernier numéro de Pelvi-périnéologie... comme certains d'entre vous avaient pu lire en son temps le dernier « édito » de Correspondances en pelvipérinéologie... Souvenirs, souvenirs...

Les dures réalités, non pas tant économiques que scientifiques et politiques, ont eu raison de cette belle revue. L'absence de lobbying convaincant a rendu impossible sa survie.

On peut le regretter, car c'est une très sympathique aventure qui se termine. Aventure avec vous, fidèles lecteurs, qui preniez plaisir, du moins nous avons la faiblesse de le croire, à nous retrouver au fil des pages, au fil des mois, au gré des articles et des dossiers... Aventure avec ce comité de rédaction toujours en productive effervescence, mais toujours redoutablement professionnel et performant...

Merci à tous ceux qui ont su s'investir.

Merci à tous les membres du comité de rédaction et en particulier à Jean-Jacques Labat.

Merci à tous les auteurs qui ont le plus souvent répondu favorablement à nos demandes parfois répétées, souvent pressantes, toujours urgentes...

Merci à nos amis de Springer qui nous ont accompagnés dans cette aventure. Merci Méline, Agnès et Nathalie, pour votre énergie et votre professionnalisme.
Merci à vous, amis lecteurs, pour la plupart membres de la Sifud, qui nous avez souvent témoigné encouragements et même, pour certains, reconnaissance...

On pourrait se moquer de la disparition de cette revue..., car de nombreuses revues existent dans la sphère de l'urologie, de la gynécologie, de la neurologie, de la rééducation, de l'imagerie, de la chirurgie... Very Frenchy, délicieusement so British ou aridement et monolithiquement American way of life... Mais par trop spécialisées, trop étroites, trop réductrices, peu éclectiques...

Pelvi-périnéologie a existé par son interdisciplinarité, par l'œcuménisme de son concept de « pelvi-périnéologie». Un souffle de nouveauté... mais mal lisible pour cette opaque officine qu'est Medline et qui voudrait bien tous nous gouverner...

Et pourquoi pas même se réjouir de cette disparition?

Quoi de plus beau que de renaître de ses cendres, de rebondir, de s'adapter, de progresser, d'être encore et d'avoir déjà été... Quel beau challenge !

Il n'est décidément pas possible de s'arrêter là !

De nouvelles idées vont sans nul doute jaillir, de nouvelles formules seront à l'évidence discutées...

Bon courage aux nouvelles équipes qui pourraient en avoir la charge.

Mon petit doigt me dit que ce n'est qu'un au revoir... Et une petite voix me susurre dans le creux de l'oreille, tout doucement chuchotée, une petite confidence : à très (très) bientôt...

G. Amarenco $(\bowtie)$

Service de neuro-urologie et d'explorations périnéales,

hôpital Tenon, 4, rue de la Chine,

F-75970 Paris cedex 20, France

e-mail : gerard.amarenco@tnn.aphp.fr 